\title{
Fourier Transform Spectroscopy Using Novel Mid-infrared Supercontinuum Sources
}

\author{
A. Khodabakhsh ${ }^{1}$, M. Nematollahi ${ }^{1}$, K. E. Jahromi ${ }^{1}$, R. Krebbers ${ }^{1}$, N. Liu ${ }^{1,2}$, M. A. Abbas ${ }^{1}$, L. Huot ${ }^{3}$, O. \\ Bang $^{4}$, F. J. M. Harren ${ }^{1}$ \\ 1 Trace Gas Research Group, Institute for Molecules and Materials, Radboud University, 6525 AJ Nijmegen, The Netherlands \\ 2 Laser Spectroscopy and Sensing Laboratory, School of Physics and Materials Science, Anhui University, 230601, Hefei, China \\ 3 NKT Photonics, Blokken 84, 3460 Birkerфd, Denmark \\ 4 NORBLIS IVS, Virumgade 35D, 2830 Virum, Denmark \\ a.khodabakhsh@science.ru.nl
}

\begin{abstract}
We present a Fourier Transform Spectrometer, especially developed for ultra-broadband mid-infrared supercontinuum sources and suitable for multispecies trace gas detection with noise equivalent detection limits in the order of 0.3-10 ppmv Hz${ }^{-1 / 2}$ for various species. $\odot 2021$ The Author(s)
\end{abstract}

\section{Introduction}

Fourier transform spectroscopy is a common and well-known technique, which is widely used in many different applications. The broad spectral coverage and fine spectral resolution offered by a Fourier transform spectrometer (FTS), specifically in the mid-infrared fingerprint region, are very crucial for gas phase multispecies detection. However, FTSs are traditionally combined with omnidirectional and low spectral brightness, incoherent thermal light sources. These sources can provide a limited interaction length with gas phase samples, which limits the detection sensitivity. In addition, the low brightness and omnidirectional emission of these sources can be problematic for some specific applications, e.g. open-path detection. Novel ultra-broadband mid-infrared supercontinuum (SC) light sources demonstrate a great potential to replace the incoherent sources in FTSs. These sources are spatially coherent and their spectral bandwidth extends to several octaves in the recent demonstrations [1]. Their spectral brightness are far more than the thermal sources and their spectral density can be engineered to provide a flat broadband spectrum, suitable for spectroscopy applications. However, compared to thermal sources, the SC sources demonstrate a high relative intensity noise (RIN) due to noise amplification in the nonlinear broadening process. By increasing the repetition rate and utilizing shorter pulse duration, the RIN of the state-of-the-art mid-infrared SC sources has been notably decreased compared to the earlier demonstrations [2]. Within the framework of the H2020 project FLAIR (Flying ultrAbroadband single-shot InfraRed sensor), we developed a home-built, compact, and transportable FTS, using only offthe-shelf optical components, especially developed to work with ultra-broadband mid-infrared SC sources for multispecies trace gas detection.

\section{Experimental setup and results}

The FTS can potentially operate in the $2-11 \mu \mathrm{m}$ bandwidth with a minimum spectral resolution of $1 \mathrm{GHz}$. In combination with a multipass cell with $32 \mathrm{~m}$ optical interaction length, the system is suitable for multispecies trace

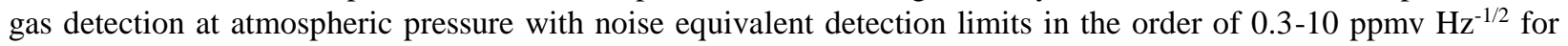
various hydrocarbons, alcohols, and aldehydes, as well as $\mathrm{CO}, \mathrm{CO}_{2}, \mathrm{NO}_{\mathrm{x}}$, and $\mathrm{SO}_{2}$. The FTS architecture is based on a Michelson interferometer, in which both of the interfering beam pairs are utilized in a balanced-detection scheme. The two out-of-phase SC interferograms are subtracted, which increases the signal by a factor of $\sim 2$ and eliminates the in-phase, common RIN of the SC source on the two interferograms. This drastically increases the SNR of the retrieved spectra. We used a stable helium-neon (He-Ne) laser, whose beam travels in parallel to the SC beam, to calibrate the optical path difference (OPD) of the FTS. We remove the DC component of the interferogram of the HeNe laser, and resample the mid-infrared SC interferogram (after balanced detection scheme) at the zero crossing positions of the He-Ne interferogram. A Fast Fourier Transform (FFT) of the OPD-calibrated SC interferogram, yields the frequency calibrated mid-infrared spectrum.

To demonstrate the versatility of our FTS, we used two different SC sources with the developed spectrometer. The first SC source is commercially available by NKT Photonics (SuperK MIR), covering the spectral bandwidth of 1.4 $4.1 \mu \mathrm{m}$ with a total average power of $\sim 450 \mathrm{~mW}$ and a repetition rate of $2.5 \mathrm{MHz}$. The second supercontinuum is developed by DTU Photonics and is based on Erbium:Ytterbium (Er:Yb) master oscillator power amplifier (MOPA) followed by cascade of different fibers [3]. This SC source covers the spectral bandwidth of $1.8-11 \mu \mathrm{m}$ with a total 
average power of $\sim 75 \mathrm{~mW}$ and a repetition rate of $3.0 \mathrm{MHz}$. Figure 1 demonstrates the measurements performed using these SC sources for trace gas detection. Panels (a) to (d) are measured using the NKT SC source and panels (e) to (g) are measured using the DTU SC source. The model spectra of $\mathrm{CH}_{4}, \mathrm{NO}, \mathrm{N}_{2} \mathrm{O}, \mathrm{SO}_{2}$ and $\mathrm{H}_{2} \mathrm{O}$ are based on the HITRAN database parameters and a Voigt profile, while the model spectra of ethyl acetate and ethane are based on the PNNL database. The concentrations as well as the spectral resolutions and averaging times are indicated in the figure caption. Note that the beam path and the box around the FTS were purged with nitrogen to minimize the water vapor concentration, whose spectrum interferes with the measurements, especially in the longer wavelength range. However, some water absorption lines are still remaining in the spectrum, e.g. see Fig 1(g).
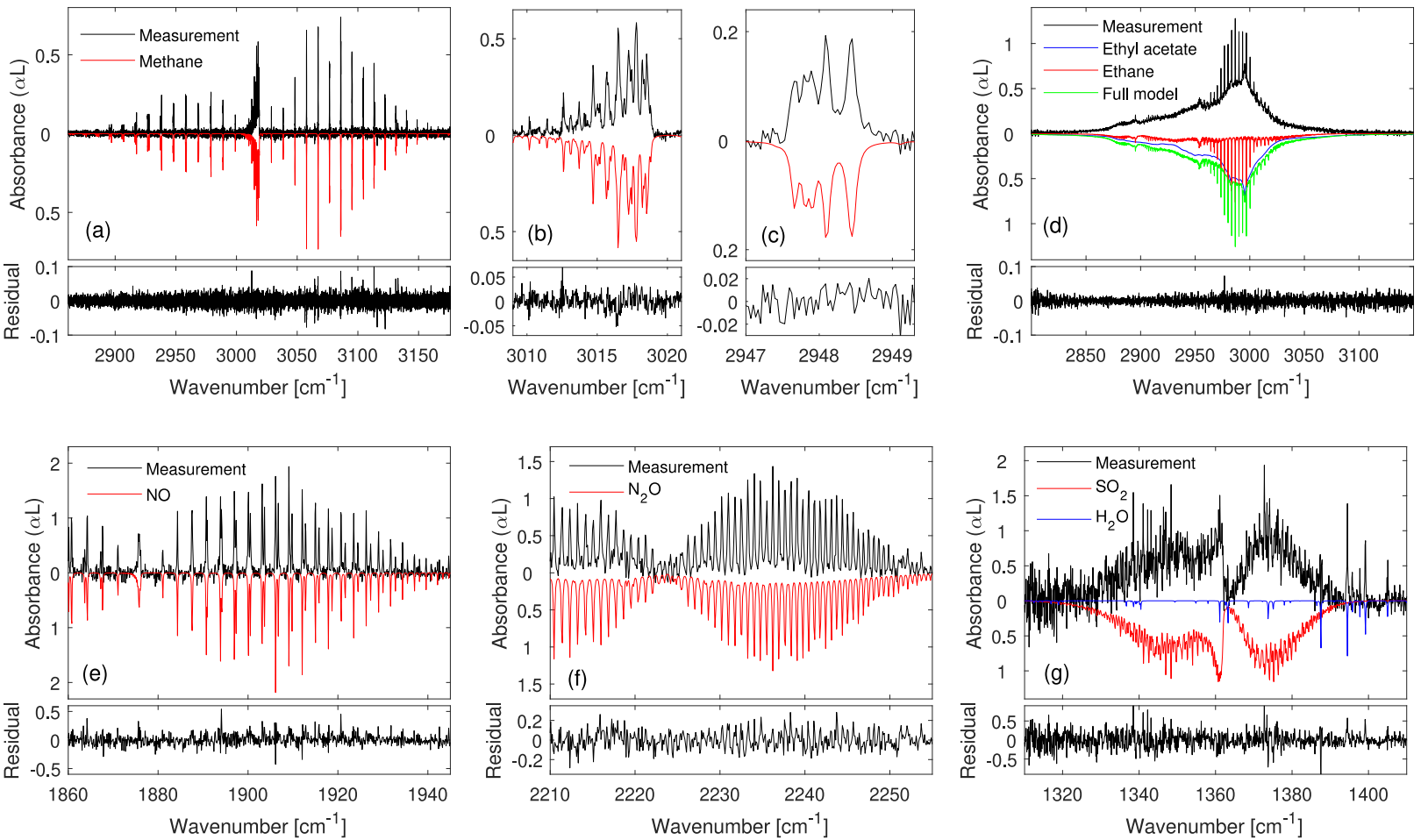

Fig. 1. Measured (in black) and modeled (in color) spectra of different species (all diluted in nitrogen) using the developed FTS and the two SC sources. The model spectra are inverted for clarity and the residual of the fits are shown in the lower panels. (a) 5 ppmv of methane (1 GHz resolution, 25 min averaging), (b) and (c) enlargements to the methane spectrum to demonstrate the quality of the fit, (d) $25 \mathrm{ppmv}$ of ethyl acetate and $5 \mathrm{ppmv}$ of ethane ( $3 \mathrm{GHz}$ resolution, 15 min averaging), (e) $40 \mathrm{ppmv}$ of $\mathrm{NO}$ (3

$\mathrm{GHz}$ resolution, $60 \mathrm{~min}$ averaging) (f) $4 \mathrm{ppmv}$ of $\mathrm{N}_{2} \mathrm{O}$ (3 GHz resolution, $60 \mathrm{~min}$ averaging, DTU SC) (g) 15 ppmv of SO 2 (3 $\mathrm{GHz}$ resolution, 60 min averaging)

Novel mid-infrared SC sources combined with an FTS, open up the possibility of broadband multispecies trace gas detection in the molecular fingerprint region, with long interaction lengths. They can potentially be used for openpath detection in the mid-infrared range, which is currently dominated by nonlinear frequency conversion of nearinfrared mode-locked lasers and optical frequency combs [4]. This can drastically reduce the complexity, cost, and size of the instrument. However, further improvements in reducing the RIN noise of these sources are still required, especially for generating longer wavelength emission using different cascaded nonlinear fibers.

\section{References}

[1] C. R. Petersen, P. M. Moselund, L. Huot, L. Hooper, and O. Bang, "Towards a table-top synchrotron based on supercontinuum generation," Infrared Phys. Technol. 91, 182-186 (2018).

[2] K. Kwarkye, M. Jensen, R. D. Engelsholm, M. K. Dasa, D. Jain, P. Bowen, P. M. Moselund, C. R. Petersen, and O. Bang, "In-amplifier and cascaded mid-infrared supercontinuum sources with low noise through gain-induced soliton spectral alignment," Scientific Reports 10, 8230 (2020).

[3] G. Woyessa, M. K. Dasa, K. Kwarkye, C. R. Petersen, and O. Bang, "Long Wavelength Mid-Infrared Supercontinuum Source," in Highbrightness Sources and Light-driven Interactions Congress 2020 (HILAS, MICS, EUVXRAY), The Optical Society, MW3C.6.

[4] G. Ycas, F. R. Giorgetta, J. T. Friedlein, D. Herman, K. C. Cossel, E. Baumann, N. R. Newbury, and I. Coddington, "Compact mid-infrared dual-comb spectrometer for outdoor spectroscopy," Opt. Express 28, 14740-14752 (2020). 